\title{
Does long-term indoor exposure to thermoneutral environments impair human body thermal acclimatization abilities?
}

\author{
Faming Wang* \\ School of Energy and Environment, Southeast University, Nanjing, 210096, China
}

*Correspondence: Prof. Faming Wang, email: dr.famingwang@gmail.com

Word count: 1,379 words (abstract, tables and references excluded)

Article type: short communication

\begin{abstract}
The goal of this short communication is to analyze a published discussion that states that longterm residing at a thermoneutral indoor temperature condition hinders human thermal acclimation capacities. According to current research, human thermal acclimation and acclimatization capacities can be easily gained through repeated heat and cold exposures mixed with physical activity over a period of days (often 3-21 days). Furthermore, heat and cold adaptations are not permanent, and heat acclimation would progressively fade away if frequent heat exposures (associated with physical work/exercise) were discontinued. People who have been heat acclimatized for a long period and live in tropical places may progressively lose their physiological and perceptual benefits when they shift to temperate zones. On the other hand, the decay of cold acclimation and cold acclimatization has not been well examined, demanding future research on this area. To summarize, there is no evidence to support the claim that extended exposure to thermoneutral conditions impairs human acclimatization abilities.
\end{abstract}

Keywords: thermal neutral; acclimation; acclimatization; adaptation; health; indoor environments 
A number of recent publications [1,2] argued that staying at an indoor neutral temperature for the majority of the time impairs human thermal acclimatization abilities, resulting in decreased body thermal resilience. If the preceding argument is correct, inactive residents living in warm and temperate regions (e.g., $C f a$ regions according to Köppen and Geiger classification [3]) should have poor thermal acclimatization abilities when compared to residents living in other climate regions around the world. To the best of the author's knowledge, there is currently no scientific evidence to support this argument.

Thermal acclimatization occurs in natural climates and is specific to the residing climate condition and level of daily physical activity [4]. Similar positive physiological adaptation can also be achieved by thermal acclimation, which can be readily developed by intentionally and consistently exposing an individual to artificial heat or cold for a specific time (varies from 5-14 days to 3-4 weeks) [5]. A thermally acclimated human body performs better at work and/or during exercise in both hot (or cold) and thermoneutral environments [6]. At present, the mechanisms of thermal acclimation induction and decay, particularly heat acclimation, are well understood.

Heat acclimation (HA) and cold acclimation (CA) are the two types of thermal acclimation (TA). HA necessitates at least 2 hours of daily heat exposure training in combination with physical activity for a minimum of 3-4 days [7]. HA can be developed using short-term, medium-term, or long-term HA training programs [8,9]. Short-term HA training lasts 4-8 days, whereas long-term HA training lasts 10-21 days [10]. Longer HA training periods may result in better heat adaptations. Females typically require more training to become completely acclimated to heat in order to establish thermoregulatory and cardiovascular stability [10]. It is widely accepted that HA-induced thermoregulatory benefits should be complete after 10 to 14 days of heat exposure [11]. Nonetheless, physiological tolerance improvements may require longer heat exposure times [12]. HA has been shown to improve the thermoregulatory system, fluid electrolytes, metabolism, the cardiovascular system, and sensory/receptor perception [13]. Table 1 tabulates the various types of adaptative changes and their demonstrated benefits.

Table 1 HA induced adaptation changes and their possible demonstrated benefits $[4,6,13]$ 


\begin{tabular}{|c|c|}
\hline $\begin{array}{l}\text { HA induced adaptation } \\
\text { changes }\end{array}$ & Improvements \\
\hline Thermoregulatory adaptations & $\begin{array}{l}\text { Reduced core temperatures during rest and work/exercise, } \\
\text { lowered skin temperatures, improved sweating capability } \\
\text { (e.g., early sweating onset), improved skin blood flow }\end{array}$ \\
\hline Fluid electrolyte adaptations & $\begin{array}{l}\text { Improved fluid balance, decreased sweating electrolyte } \\
\text { losses, increased body plasma volume }\end{array}$ \\
\hline Metabolic adaptations & $\begin{array}{l}\text { Decreased metabolic rate, decreased lactate threshold \& } \\
\text { muscle lactate, increased skeletal muscle force generation }\end{array}$ \\
\hline Cardiovascular adaptations & $\begin{array}{l}\text { Increased stroke volume \& cardiac output, enhanced cardiac } \\
\text { efficiency \& cardiac pressure generation, reduced heart rate }\end{array}$ \\
\hline $\begin{array}{l}\text { Thermoreceptor sensory } \\
\text { adaptations }\end{array}$ & $\begin{array}{l}\text { Reduced thermal sensation, improved thermal discomfort, } \\
\text { improved exertion perception }\end{array}$ \\
\hline Molecular adaptation & $\begin{array}{l}\text { Increased heat shock protein (HSP) levels, altered HSP } \\
\text { expression patterns. }\end{array}$ \\
\hline
\end{tabular}

The thermoregulation and metabolic adaptations shown in Table 1 can significantly improve the heat transfer from the core of the body to the peripheral skin, alleviating thermal strain in hot environments and thereby improving work and exercise performance [14].

In recent decades, cold acclimation (CA) and cold acclimatization (CAT) have received far less attention than heat adaptation. CA is an adaptation to a single stimulus, such as cold temperatures. Cold acclimatization occurs naturally as a result of living and working in cold regions. CAT reflects a variety of stimuli, including cold temperatures. People living in the circumpolar region, for example, are not only subjected to cold temperatures, but their diet and daytime length differ significantly from those living in other regions of the world [15-17]. There are three known types of cold acclimatization: habituation, local acclimatization, and whole-body acclimatization. Habituation can be developed by exposing the body to short-term severe cold. Habitation improves both perceptual and physiological responses to cold. Noticeable perceptual benefits may include reduced thermal sensation, thermal discomfort, and pain sensation, while physiological benefits may be demonstrated by reduced blood pressure, decreased plasma 
concentration of noradrenaline, reduced shivering, reduced metabolic rate, reduced heart rate, and reduced respiratory rate. Local acclimatization is often developed in human hands. Better blood circulation in the hands is often observed among locally cold acclimatized people. Meanwhile, the phenomenon of cold-induced vasoconstriction in locally acclimatized people is weaker than that in unacclimatized people. Whole-body CA can be developed in as little as 10 days [15]. Unlike heat adaptation, which results in a fairly uniform thermoregulatory changing pattern across individuals from different regions, whole-body CA results in four types of adaptation depending on the severity of cold stress and personal factors such as diet, fitness, and body fat content [18, 19]: metabolic, hypothermic, insulative and insulative-hypothermic acclimation. The characteristics of these four distinct adaptation patterns are shown in Table 2.

Table 2 Whole-body cold adaptation patterns and their characteristics [17-23]

\section{Cold adaptation patterns Characteristics}

Metabolic adaptation Unchanged core temperature, increased skin temperature, increased metabolic rate, enhanced shivering thermogenesis \& non-shivering thermogenesis (found in Alacaluf Indians and Eskimos)

Hypothermic adaptation

Decreased core temperature, decreased metabolic rate, unchanged skin temperature (found in bushmen of the Kalahari Desert and Peruvian Indians)

Insulative adaptation Unchanged core temperature, unchanged metabolic rate, decreased skin temperature, enhanced vasoconstriction, improved muscle blood slow redistribution (body heat towards the subcutaneous shell) (found in coastal aborigines of tropical northern Australia)

Insulative-hypothermic Unchanged metabolic rate, decreased skin temperatures, decreased adaptation core temperature, sustained cutaneous vasoconstriction reaction (found in central Australian aborigines, nomadic Lapps, and Korean \& Japanese women divers) 
Whole-body cold water immersion has been widely used to develop the four cold adaptation patterns depicted in Table 2. Hypothermic cold acclimation can be achieved in 9 days by immersing the entire body in cold water at $15^{\circ} \mathrm{C}$ for $20-60$ min per day. Insulative cold acclimation may be achieved by immersing the entire human body in $18{ }^{\circ} \mathrm{C}$ cold water for $90 \mathrm{~min}$ per day, five days per week, for a total of five weeks. However, the cold exposure programs described above may not always result in the same pattern of cold adaptation in different ethnic groups. The exact factors influencing cold adaptation patterns induced by chronic cold exposure are still unknown. Cold acclimation types may be influenced by the intensity and extent of body cooling, cold exposure frequency and duration, and individual factors such as body composition and physical fitness $[20,21]$.

Adaptations to heat and cold are not permanent. The decay of heat acclimation has been extensively investigated [12, 22-26]. First, heat acclimation would gradually fade away if the repeated heat exposures (combined with physical work/exercise) are discontinued. The physiological and perceptual benefits of heat acclimation may last for about a week before fading, with approximately $75 \%$ lost within 4-6 days or three weeks of the heat exposure ceasing [12,26]. In a systematic review and meta-analysis conducted by Daanen et al. [25], a 2.5\% daily decay in heart rate and core temperatures was deduced from reported literature studies. Thus, the HAinduced physiological and perceptual benefits are expected to disappear after about 40 days of no heat exposure. Though the number of HA training days had no effect on the decay in heat adaptations, it did have an effect on sweating rate decay. That is, the longer the HA period, the less sweating rate decay could be observed [26]. On the other hand, re-acclimation during the decay period occurs faster than initial heat acclimation. Furthermore, exposing for a day or two in cool temperature conditions would not interfere with HA. Nonetheless, exposure to cold conditions for over 4 days may hasten the decay of HA [27]. Furthermore, after achieving HA, training and HA can be alternated every second or third day [7]. Long-term heat acclimatized people living in tropical regions may gradually lose their physiological and perceptual benefits (heat dissipation response, thermoregulatory responses, body fluid regulation functions, etc.) when they migrate to temperate regions [28-32]. Heat tolerance gained from long-term heat acclimatization also decreased gradually after relocating to a temperate region, confirming that long-term heat acclimatization is a partially phenotypic phenomenon [32]. Some of the benefits may last longer 
than four years, though some aspects of heat adaptation may have decayed significantly in a short period of time [31]. To date, it is unknown how long it takes for an individual to regain complete heat acclimatization after returning from temperate regions to tropical regions where they were born and raised.

There is currently no information available on the decay of cold acclimation and cold acclimatization, necessitating further investigation. Jones et al. [33] investigated the effects of cold acclimation on physiological and perceptual responses while working in hot conditions. The findings suggest that cold acclimatization ability is unlikely to be associated with an increased risk of thermal strain when subjected to physical activity in hot environments, implying that cold acclimation does not pose a higher risk of hyperthermia during subsequent work and exercise in hot environments.

Based on reported results of preceding studies [4-33], it is safe to conclude that thermal acclimation can be easily obtained through heat/cold exposures combined with physical activity training. If an individual is healthy, staying in thermoneutral indoor and outdoor environments for an extended period of time should not affect thermal acclimatization (acclimation) abilities. Physical, genetic and behavioral traits have all been rigorously tested and well evolved over the long period of human evolution. Human thermal adaptation (morphological, chemical, functional, and genetic alterations that may aid the body in mitigating thermophysiological strain when exposed to thermal stress) [4] and thermal tolerance (the ability of an organism to survive a normally lethal thermal stress) [34] abilities are far more powerful than previously thought. In conclusion, there is no scientific evidence to support the argument that staying in a neutral temperature most of the time impedes human thermal acclimatization abilities, resulting in decreased thermal resilience.

\section{Declaration of Interest Statement}

I wish to confirm that there are no known conflicts of interest associated with this short communication. 


\section{References}

[1] W. Luo, R. Kramer, Y. de Kort, W. van Marken Lichtenbelt, Effectiveness of personal comfort systems on whole-body thermal comfort-a systematic review on which body segments to target, Energ Build 256 (2022) 111766.

[2] W. Luo, R. Kramer, Y. de Kort, P. Rense, W. van Marken Lichtenbelt, The efects of a novel personal comfort system on thermal comfort, physiology and perceived indoor environmental quality, and its health implications-Stimulating human thermoregulation without compromising thermal comfort, Indoor Air 32 (1) (2022) e12951.

[3] H.E. Beck, N.E. Zimmermann, T.R. McVicar, N. Vergopolan, A. Berg, E.F. Wood, Present and future Köppen-Geiger climate classification maps at 1-km resolution, Sci Data 5 (2018) 180214.

[4] N.A.S. Taylor, Human heat adaptation, Compr Physiol 4 (2014) 325-365.

[5] C.L. Benjamin, Y. Sekiguchi, L.A. Fry, D.J. Casa, Performance changes following heat acclimation and the factors that influence these changes: meta-analysis and meta-regression, Front Physiol 10 (2019) 1448.

[6] M.N. Sawka, L.R. Leon, S.J. Montain, L.A. Sonna, Integrated physiological mechanisms of exercise performance, adaptation, and maladaptation to heat stress, Compr Physiol 1 (2011) 1883-1928.

[7] J.D. Périard, S. Racinais, M.N. Sawka, Adaptation and mechanisms of human heat acclimation: applications for competitive athletes and sports, Scand J Med Sci Sports 25 (S1) (2015) 20-38.

[8] J.N. Moss, F.M. Bayne, F. Castelli, M.R. Naughton, T.C. Reeve, S.J. Trangmar, R.W.A. Mackenzie, C.J. Tyler, Short-term isothermic heat acclimation elicits beneficial adaptations but medium-term elicits a more complete adaptation, Eur J Appl Physiol 120 (1) (2020) 243254.

[9] J.R. Casadio, A.E. Kilding, J.D. Cotter, P.B. Laursen, From lab to real world: heat acclimation considerations for elite athletes, Sports Med 47 (2017) 1467-1476.

[10] J.A. Mee, O.R. Gibson, J. Doust, N.S. Maxwell, A comparison of males and females' temporal patterning to short- and long-term heat acclimation, Scand J Med Sci Sports 25 (S1) (2015) 250-258. 
[11] H.A.M. Daanen, J.A. Herweijer, Effectiveness of an indoor preparation program to increase thermal resilience in elderly for heat waves, Build Environ 83 (2015) 115-119.

[12] M.N. Sawka, J.D. Périard, S. Racinais, Heat acclimatization to improve athletic performance in warm-hot environments, Sports Sci Exchang 28 (153) (2015) 1-6.

[13] C.J. Tyler, T. Reeve, G.J. Hodges, S.S. Cheung, The effect of heat adaptation on physiology, perception and exercise performance in the heat: a meta-analysis, Sports Med 46 (11) (2016) 1699-1724.

[14] Schlader ZJ, Mündel T. Heat acclimation, in: Mooren FC (ed), Encyclopedia of Exercise Medicine in Health and Disease, Springer-Verlag, Berlin, Germany, 2012.

[15] H. Rintamäki, Human cold acclimatisation and acclimation, Int J Circumpolar Health 60 (3) (2001) 422-429.

[16] H.A.M. Daanen, W.D. van Marken Lichtenbelt, Human whole body cold adaptation, Temperature 3 (1) (2016) 104-118.

[17] Young AJ. Human adaptation to cold stress, in: Shiraki K, Sagawa S, Yousef MK (eds) Physiological Basis of Occupational Health: Stressful Environments, SPB Academic Publishing BV, Amsterdam, The Netherlands, 1996.

[18] Tipton MJ, Pandolf KB, Sawka MN, Werner J, Taylor NAS. Physiological adaptation to hot and cold environments, in: Taylor NAS, Gronllier H (eds), Physiological Bases of Human Performance During Work and Exercise, Churchill Livingstone Publishing, London, UK, 2008.

[19] M. Brazaitis, N. Eimantas, L. Daniuseviciute, N. Baranauskiene, E. Skrodeniene, A. Skurvydas, Time course of physiological and psychological responses in humans during a 20dau severe-cold-acclimation programme, PLoS One 9 (4) (2014) e94698.

[20] D.P. Blondin, A. Daoud, T. Taylor, H.C. Tingelstad, V. Bézaire, D. Richard, A.C. Carpentier, A.W. Taylor, M.E. Harper, C. Aguer, F. Haman, Four-week cold acclimation in adult humans shifts uncoupling thermogenesis from skeletal muscles to brown adipose tissue, J Physiol 595 (6) (2017) 2099-2113.

[21] J.H.M. Bittel, Heat debt as an index for cold adaptation in men, J Appl Physiol 62 (1987) 1627-1634.

[22] A.A.J.J. Van der Lans, J. Koeks, B. Brans, G.H.E. Vijgen, M.G.W. Visser, M.J. Vosselman, J. Hansen, J.A. Jörgensen, F.M. Mottaghy, P. Schrauwen, W.D. van Marken Lichtenbelt, Cold 
acclimation recruits human brown fat and increases nonshivering thermogenesis, J Clin Invest 123 (8) (2013) 3395-3403.

[23] K. Gordon, D.P. Blondin, B.J. Friesen, H.C. Tingelstad, G.P. Kenny, F. Haman, Seven days of cold acclimation substantially reduces shivering intensity and increases nonshivering thermogenesis in adult humans, J Appl Physiol 126 (6) (2019) 1598-1606.

[24] A.T. Garrett, N.J. Rehrer, M.L. Patterson, Induction and decay of short-term heat acclimation in moderately and highly trained athletes, Sports Med 41 (9) (2011) 757-771.

[25] H.A.M. Daanen, S. Racinais, J.D. Périard, Heat acclimation decay and re-induction: a systematic review and meta-analysis, Sports Med 48 (2) (2018) 409-430.

[26] K.B. Pandolf, Time course of heat acclimation and its decay, Int J Sports Med 19 (Suppl 2) (1998) S157-S160.

[27] M. Saat, R.G. Sirisinghe, R. Singh, Y. Tochihara, Decay of heat acclimation during exercise in cold and exposure to cold environment, Eur J Appl Physiol 95 (2005) 313-320.

[28] H. Wakabayashi, T. Wijayanto, J.Y. Lee, N. Hashiguchi, M. Saat, Y. Tochihara, Comparison of heat dissipation response between Malaysian and Japanese males during exercise in humid heat stress, Int J Biometeorol 55 (4) (2011) 509-517.

[29] H. Wakabayashi, T. Wijayanto, J.Y. Lee, N. Hashiguchi, M. Saat, Y. Tochihara, Comparison of thermoregulatory responses to heat between Malaysian and Japanese males during leg immersion, Int J Biometeorol 55 (4) (2011) 491-500.

[30] H. Wakabayashi, T. Wijayanto, J.Y. Lee, N. Hashiguchi, M. Saat, Y. Tochihara, A comparison of hydration effect on body fluid and temperature regulation between Malaysian and Japanese males exercising at mild dehydration in humid heat, J Physiol Anthropol 33 (2014) 5 .

[31] T. Wijayanto, H. Wakabayashi, S. Toramoto, Y. Tochihara, Effects of duration of stay in temperate area on thermoregulatory responses to passive heat exposure in tropical south-east Asian males residing in Japan, J Physiol Anthropol 31 (2012) 25.

[32] J.B. Lee, J.S. Bae, M.Y. Lee, H.M. Yang, Y.K. Min, H.Y. Song, K.K. Ko, J.T. Kwon, T. Matsumoto, The change in peripheral sweating mechanisms of the tropical Malaysian who stays in Japan, J Therm Biol 29 (7-8) (2004) 743-747. 
[33] D.M. Jones, K.A. Rostomily, C.M. Pautz, D.W. Ito, S.P. Bailey, B. Roelands, M.J. Buono, R. Meeusen, Cold acclimation does not alter physiological or perceptual responses during subsequent exercise in the heat, Mil Med 182 (9-10) (2017) e1958-e1964.

[34] L.B. Buckley, R.B. Huey, How extreme temperatures impact organisms and the evolution of their thermal tolerance, Integr Comp Biol 56 (1) (2016) 98-109. 